\title{
INFLUENCE OF THE INDUCED AIRFLOW ON CALENDERING
}

\author{
Karel ADÁMEK, Jan KOLÁŘ
}

\begin{abstract}
The article presents the seeking of possible correlation between the usable characteristics of a treated textile layer, finished by calendering process and the flow field arising by the rotation of calendering drums in the undisturbed surroundings. Thereby the methods of numerical flow simulation were used here.
\end{abstract}

\section{INTRODUCTION}

The contribution describes the flows arising by the rotation of calendering drums in the unmoved surroundings. It is practically impossible to measure some characteristics of such flow field, above all in the contact area of a pair of rotating calendering drums. Therefore, the methods of numerical flow simulations were used for the detailed description of the flow field, first of all of components of the velocity vector, further of the both static and dynamic pressure etc. The presented results include both the global models of rotating drums and the detailed models of flows in small gaps among elementary protrusions on the surface of the drum.

\section{Global Model}

It is well known that the rotating surface creates in the unmoved surroundings a complicated flow field. In addition, here it is presented the influence of the very air permeable textile layer which transports an indispensable flow volume in the contact area between the drums. For the same geometry and mesh of the model, three different boundary conditions were used as follows:

- Rotating drums with the incoming textile layer

- Furthermore, the drums are heated

- Furthermore, the layer permeability is defined.

The defined drums peripheral velocity of $15 \mathrm{~m} / \mathrm{s}$ is identical with the transport velocity of the textile layer. Due to the very small gaps among the elementary protrusions of the drum surface, the mesh must be very fine. But due to the limited capacity of the working memory it is not possible to model the whole periphery of drums with all elementary protrusions. Instead, some global roughness is defined only and the simple twodimensional model is used.

The results of all solved cases are very similar; therefore, due to place saving, only one typical case is presented here.

Fig. 1 shows the streamlines of the induced flow around the rotating drums. Different colours present the origin of streamlines, for instance - green = movement of the upper drum, red = movement of the lower drum, blue = air inlet from the left surroundings, etc.

\footnotetext{
• Karel Adámek, Jan Kolář, VúTS Liberec a.S., U Jezu 525/4, P.O.B. 92, CZ-46119 Liberec 1, karel.adamek@vuts.cz, jan.kolar@vuts.cz
} 

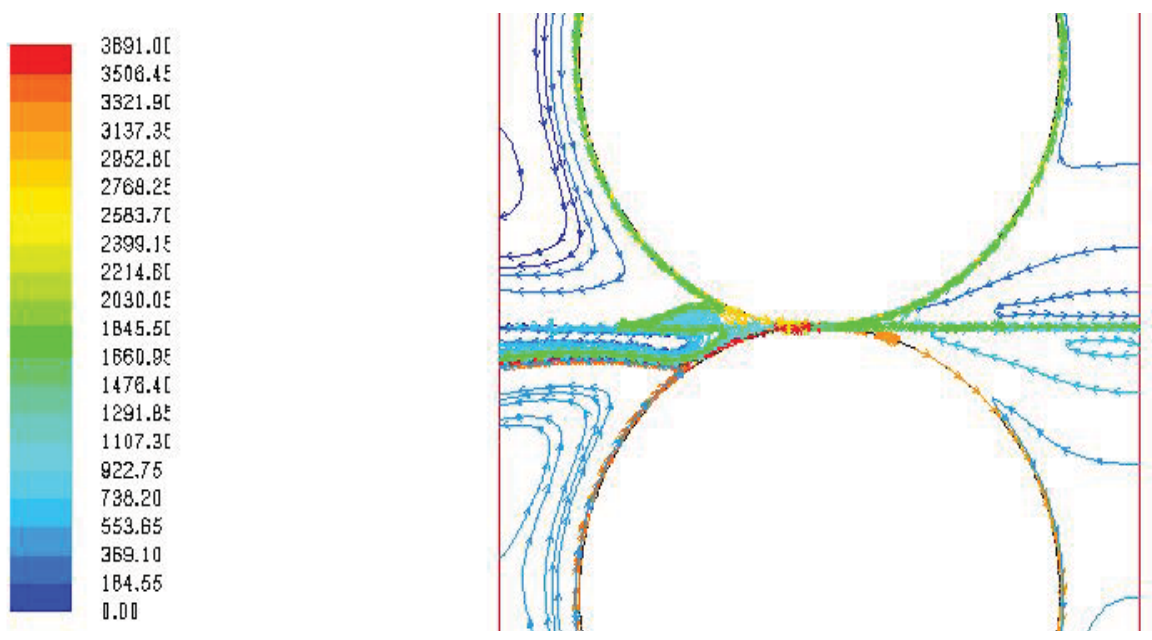

Figure 1: Streamlines

Fig. 2 presents the velocity isolines and Fig. 3 the corresponding pressure isolines. It is visible that on the inlet side (here from the left), two streams are flowing to one another along both rotating surfaces and they collide in the inlet wedge. There arises some overpressure and then some air volumes, incoming by the drums rotation, are flowing back in the horizontal plane to the left - against the movement of the supplied material.
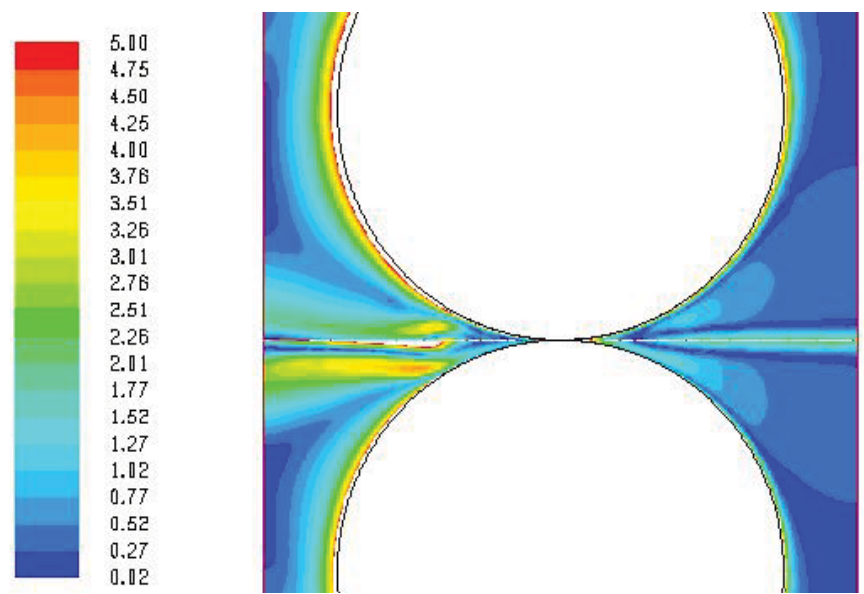

Figure 2: Velocity field

The lower drum pulls a higher air volume because of its higher roughness, comparing with the smooth upper drum. Therefore, the horizontal backflow from the inlet wedge is higher than that in the lower part.

On the outlet side (on the right), both rotating drums pull some air volume, too, in the direction of the rotation (apart). Therefore, in the outlet wedge, some underpressure arises and an air volume is sucked here from the surroundings (from the right). The mixing effect of both flows is well visible in the temperature field when the drums are heated. Fig. 3 shows that the area of the increased temperature in the inlet wedge is "flowing" back to the left, partially disturbed by cold flows around rotating drums and in 
the treated layer, too. In the outlet wedge there arises a wide area of increased temperature, too, further the temperature decreases due to suction of the cold air from the surroundings.
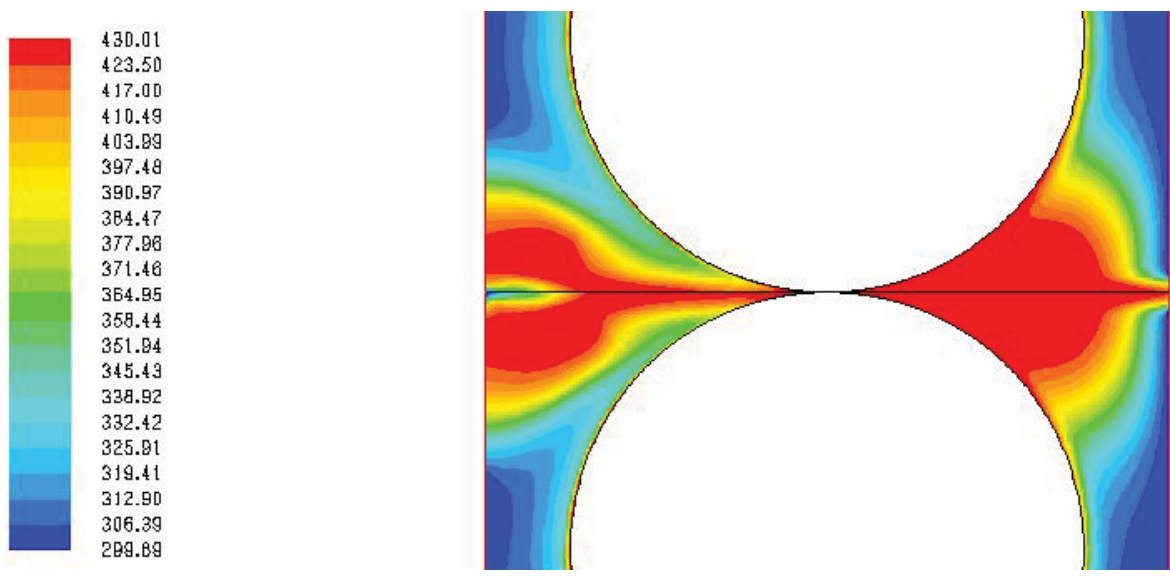

Figure 3: Temperature field between the drums

The details of the pressure field in the contact area of drums are not presented here due to a very small gap between both drums. The better view is given with the following pressure profile in the horizontal plane between the drums whose typical result is shown in Fig. 4. Before the contact point, the pressure is increasing in the correspondence with the air compression in the inlet wedge, and then it falls down in the underpressure due to air suction by rotating drums from the surrounding and finally the pressure increases again on the value of the atmospheric pressure.

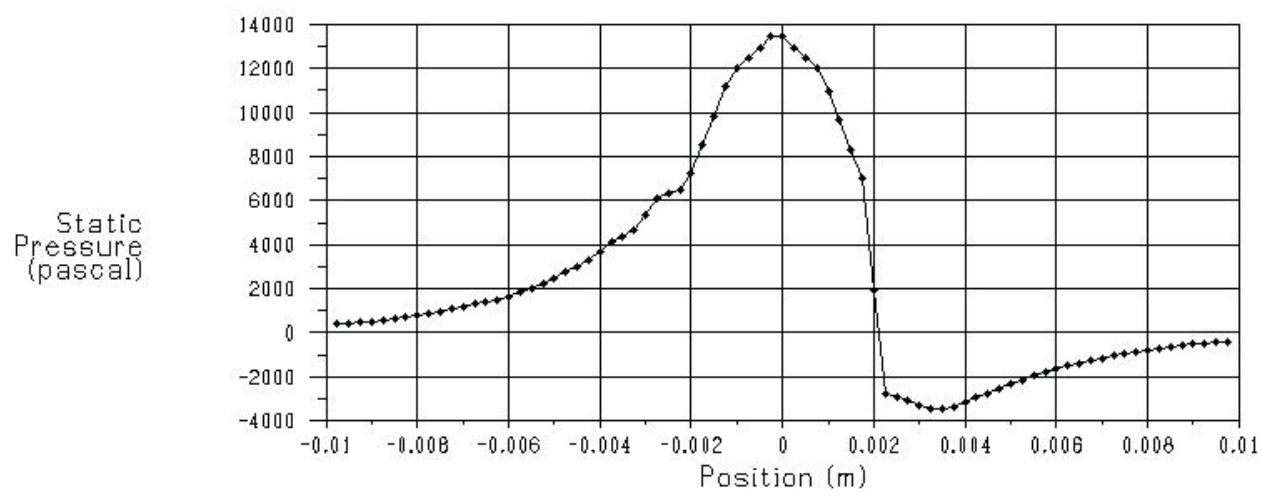

Figure 4: Pressure profile between the drums

The velocity profile is not presented here. It is practically constant because the discussed model contains the transported textile layer as an air layer of a transporting velocity of $15 \mathrm{~m} / \mathrm{s}$. Such simplification is acceptable because the inserting layer is very "airy", the layer volume contains $20 \%$ of textiles only.

As a partial conclusion we can state that in the contact area of rotating drums there arises not only a pressing force but also an increased aerodynamic influence. This global 
influence amplifies the elementary aerodynamic forces which are depicted hereinafter in Paragraph 3.

\section{Detailed Model}

The real "roughness" of the rotating drums induces the local velocity field which interacts with the global flow according to Paragraph 2. For a complex flow field simulation it is necessary to prepare and solve the complex three-dimensional model, but its scope is very large.

Here are presented the main results of one case of numerical simulation of detailed flow field among the elementary protrusions situated on the drum surface. They are the results of the interaction between the immovable surroundings and small protrusions, fixed on the moving drum surface of large diameter as to Fig. 5. The typical flow field is shown in Fgs. 6 to 8 as the velocity components in the contact plane - on the tips of the elementary protrusions.
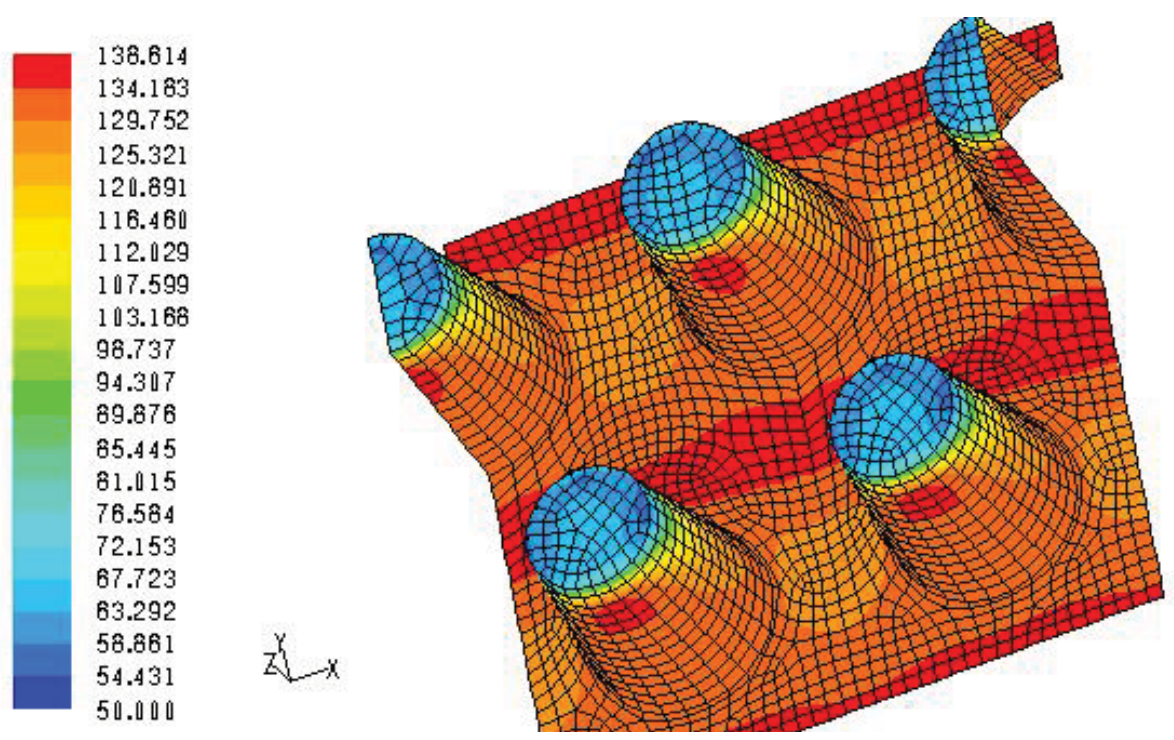

Figure 5: Typical pattern of contact protrusions - mesh, coloured by the dynamic pressure values

The method of moving mesh gives the flow field of air movement, arising through the rotation of the drum surface. The elementary protrusions, situated on the rotating surface, interact with the air in surroundings above all through the pressure and viscose forces. The direction of the rotation is here $(+y)$, the main component $(y)$ as shown in Fig. 6 should have $15 \mathrm{~m} / \mathrm{s}$, but in the gaps among the individual protrusions, the velocity value is smaller. Of course, in a shorter distance from the drum surface, the velocity increases to $15 \mathrm{~m} / \mathrm{s}$ and becomes more uniform. 

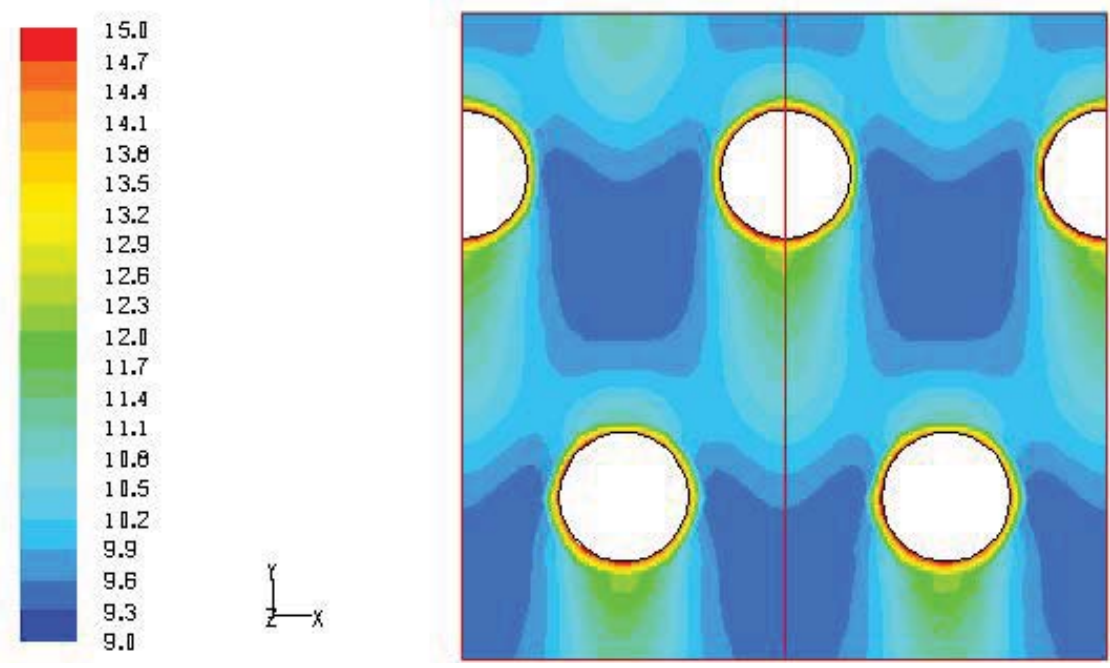

Figure 6: Main velocity component $(y)$ in the contact plane
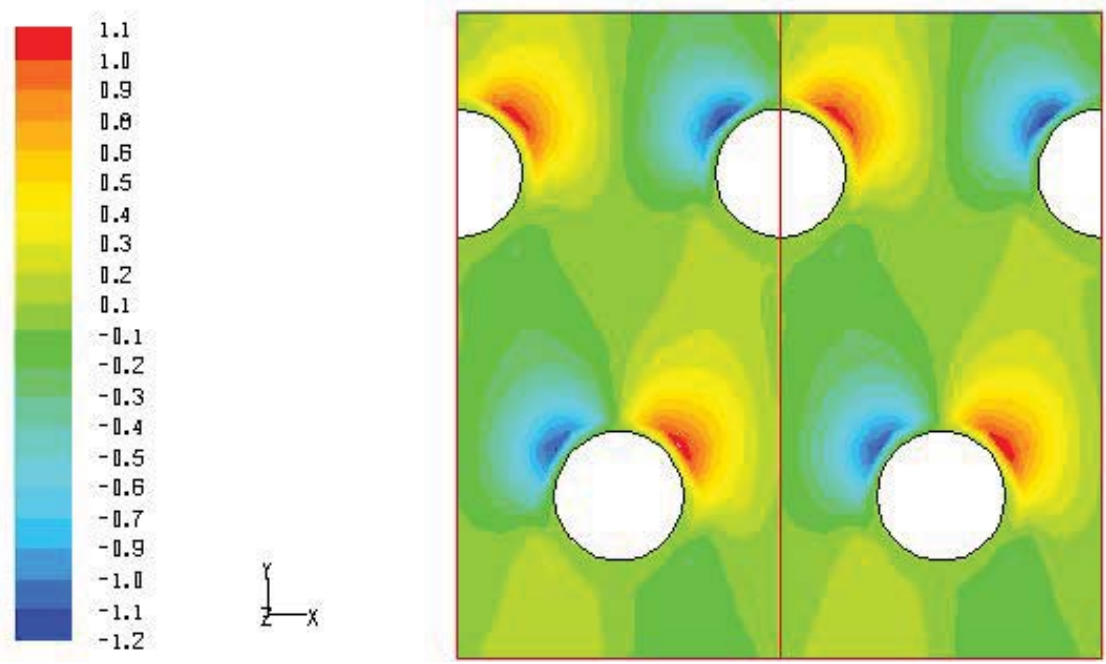

Figure 7: Cross velocity component $(x)$ in the contact plane

Fig. 7 shows a cross flow ( $x$ - alternates to left and right) and Fig. 8 shows the radial flow ( $z$ - alternates up and down), both according to the flow around the protrusions. The dynamic pressure of those components can have a certain influence on the treated textile layer. 

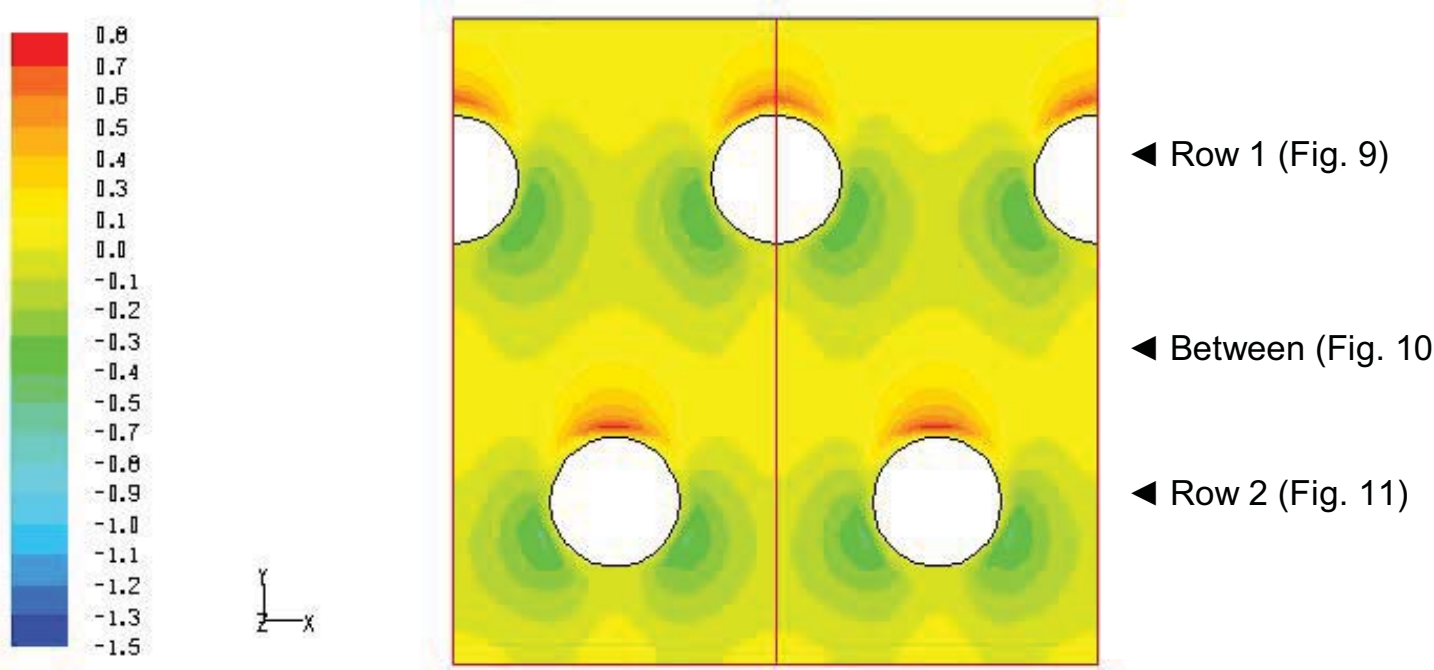

Figure 8: Radial velocity component $(z)$ upright to the contact plane
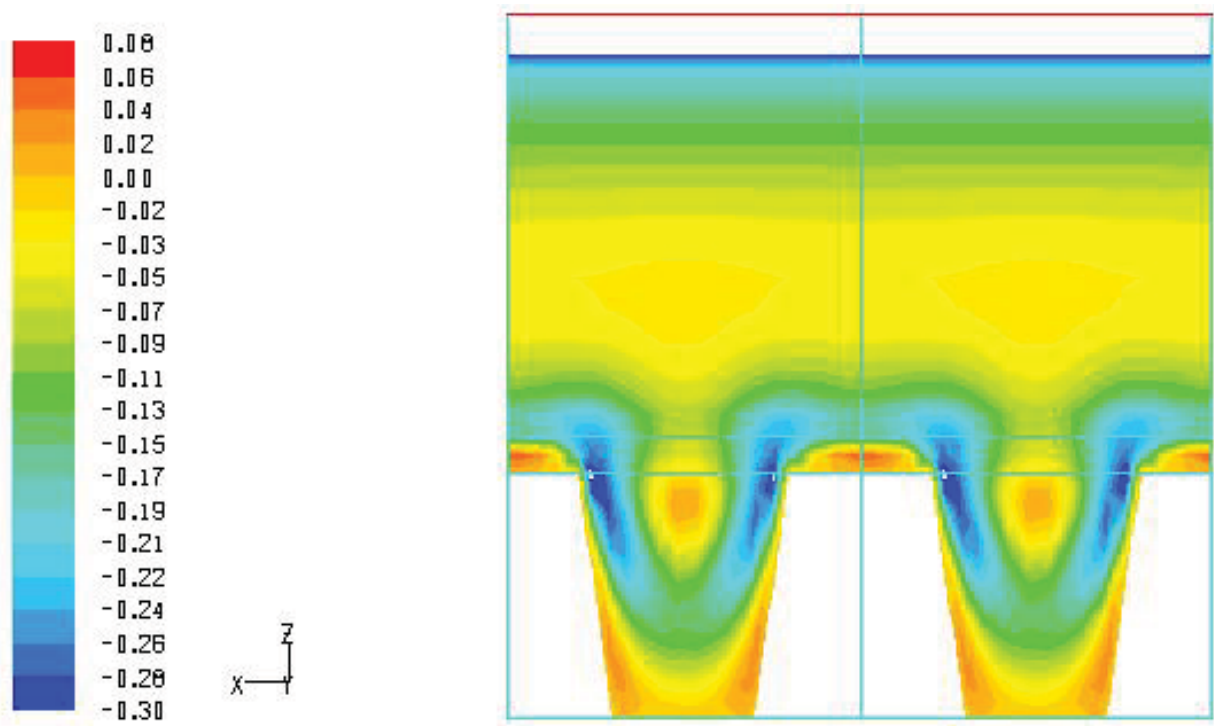

Figure 9: Radial velocity component $(z)$ - cross section through the protrusions (row 1 )

The possible mixing movements in the flow field are shown for instance in the serial of Fig. 9 to Fig. 11 with radial velocity in several cross sections in the planes $x-z$ (across to the direction of the rotation). Without any next analysis, it is visible the air movement in this direction - the details depend on the actual position $(y)$ of the plane of observation $(y-z)$. 

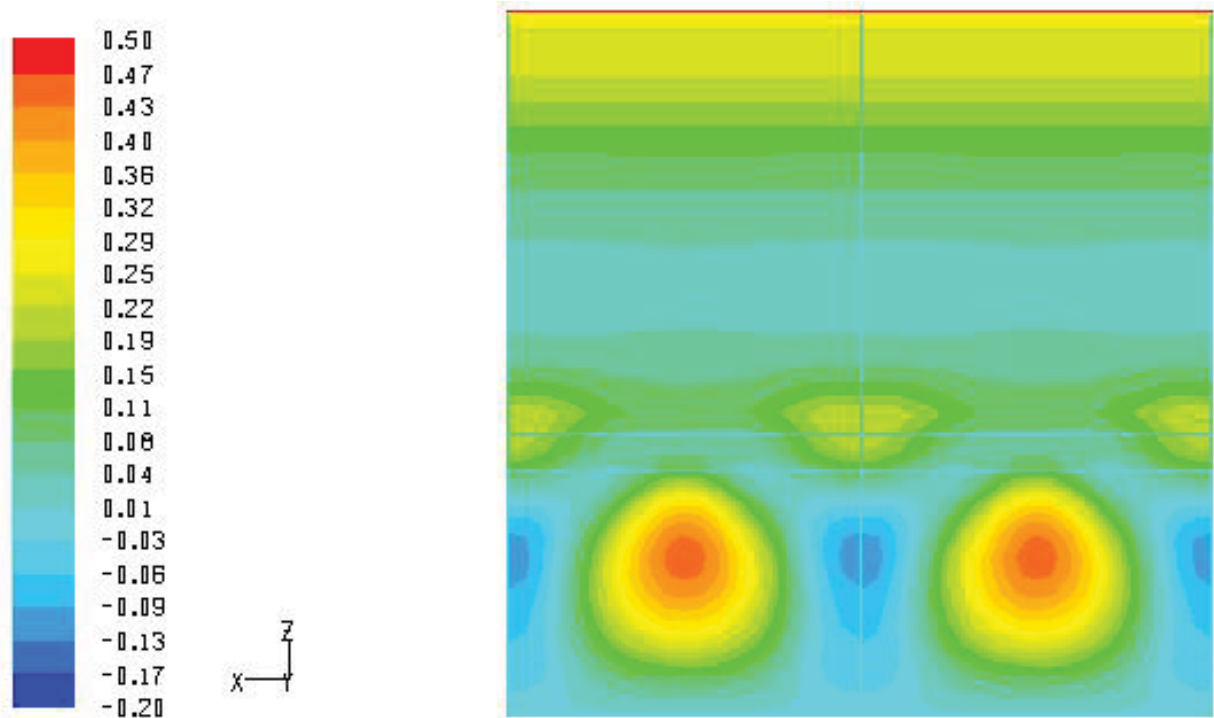

Figure 10: Radial velocity component $(z)$ - cross section between the protrusions
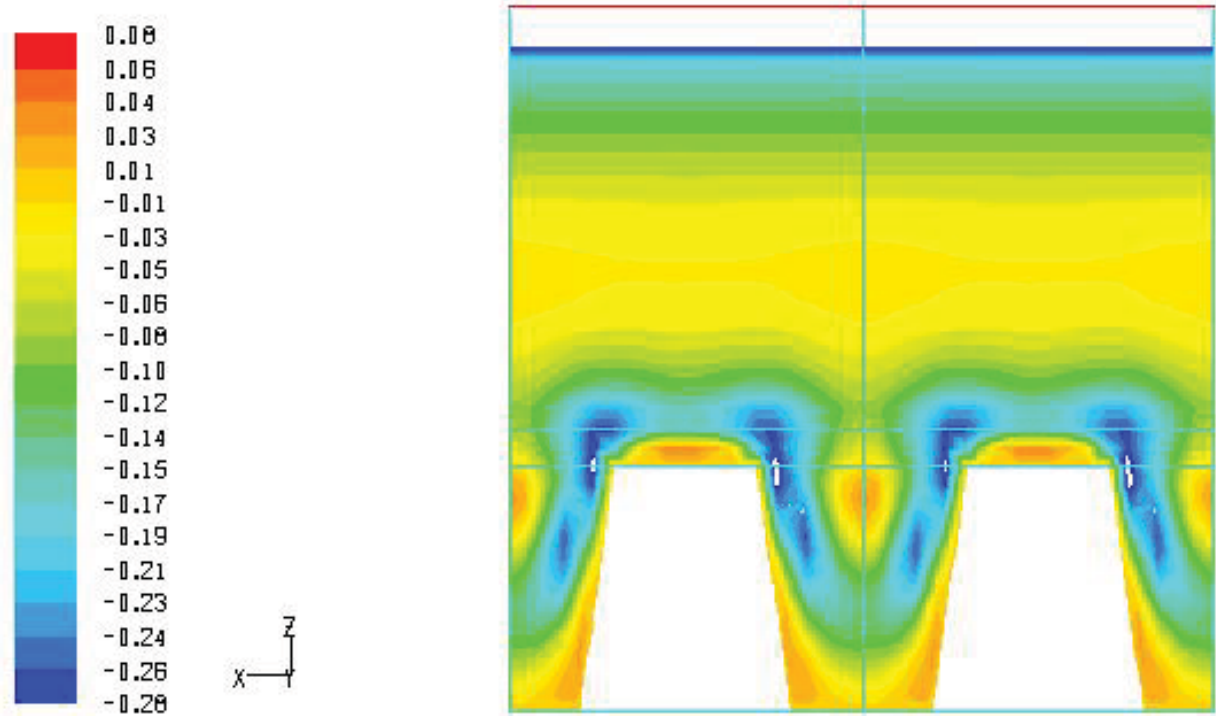

Figure 11: Radial velocity component $(z)$ - cross section through the protrusions (row 2 )

As the conclusion we can state that the flow field among the elementary protrusions, fixed on the rotating drum surface, is characterized by actual components of the velocity vector. The arising dynamic forces can have some influence on the processed textile layer and on its elementary fibres, too. In such a way, they could have an influence on their usable characteristics, too. 


\section{SUMMARY}

The article presents some results of numerical flow simulations around two rotating drums, one of them furnished by small protrusions. Along such surface there arises the complicated flow field whose dynamic pressure could have some influences on the processed material. And more, the influences could be amplified by the global influence of the overpressure on the inlet side between two jointly rotating drums.

Of course, a complex model could give a better image about the flow field, but the mesh of very fine (small) protrusions fixed on the large drum diameter must be very fine, too, and for such a complex model the actual working memory is not sufficient.

The contact area of calendering drums is not accessible for any measuring sensors that is why the method of numerical flow simulation is the sole possibility for the description of the flow field arising by the rotation of such two drums.

The velocity components or their dynamic pressure create some force impact on the processed material. 